$\xi=-1$

\title{
Support vector machine in the anticipation of currency markets
}

\author{
V. Lalithendra Nadh ${ }^{1 *}$, G. Syam Prasad ${ }^{2}$ \\ ${ }^{1}$ Sree Vidyanikethan Engineering College (Autonomous), Tirupati, India \\ ${ }^{2}$ K L E F (Deemed to be University), Green Fields, Vaddeswaram, India \\ *Email: lalithendravenigalla@gmail.com
}

\begin{abstract}
Various researchers have done an expansive research within the domain of stock market anticipation. The majority of the anticipated models is confronting some pivotal troubles because of the likelihood of the market. Numerous normal models are accurate when the data is linear. In any case, the expectation in view of nonlinear data could be a testing movement. From past twenty years with the progression of innovation and the artificial intelligence, including machine learning approaches like a Support Vector Machine it becomes conceivable to estimate in light of nonlinear data. Modern researchers are combining GA (Genetic Algorithm) with SVM to achieve highly precise outcomes. This analysis compares the SVM and ESVM with other conventional models and other machine learning methods in the domain of currency market prediction. Finally, the consequence of SVM when compared with different models it is demonstrated that SVM is the premier for foreseeing.
\end{abstract}

Keywords: Back Propagation Neural Networks (BPNN), Support Vector Machine (SVM), Support Vector Regression (SVR)

\section{Introduction}

Stock market anticipation is a testing assignment for specialists in view of its crucial nature. Consistently, many individuals in the world are making stock market investments. Foreseeing the exact stock patterns and costs becomes a major task in stock market speculations. The stock patterns straightforwardly or indirectly rely upon numerous things like news, peoples' sentiment, political issues, and so forth. So it is not so natural to foresee the stock prices just by taking historical data into consideration. In spite of the fact that Prediction of the stock price is a tough task, it is essential for making better decisions, to limit the speculation hazards and to accomplish high benefits with least capital. There are such a large number of traditional predictive models till now like Autoregressive integrated moving average (ARIMA) which is an improvement of Autoregressive moving average (ARMA). For the past two decades, researchers have completely focused on machine learning approaches for the forecast of stock prices like artificial neural networks, backpropagation neural networks. Predicting linear time series data is an easy task compared to nonlinear time series data. Among the entire machine learning approaches Support vector machine is giving more exact outcomes when contrasted with other machine learning approaches mainly in terms of nonlinear time series data.

Support Vector Machine (SVM) is a supervised machine learning algorithm presented by Vapnik in the year 1995. SVM is totally different from other conventional forecast approaches. SVM is basically utilized for classification of objects, pattern recognition, and regression analysis. Structural risk minimization is the fundamental standard of SVM though the other machine learning approaches are based on empirical risk minimization principle. The SVM will diminish the miss- classification error of the arrangement of previously trained data and then estimates the function of reducing the upper bound of generalization. Without determining weights the networks of the training data, it gets geometric attributes of feature space and isolates the optimal arrangement with the smallest training set. The SVM has the ability to achieve high generalization performance. The conventional methodologies or the other machine learning approaches require a huge training data for input pattern estimation and because of its overfitting nature generalizing the results is extremely troublesome. The SVM has the best execution; Based on the execution of classification and the ability of classifier's improvement is affected by its number of feature variables or by its dimension. This Work gives an examination of the how a support vector machine functions better when contrasted with other machine learning approaches.

\section{Literature Review}

(PrasadDas \& Padhy, 2012) Support vector machine in fig 2 and the Backpropagation neural networks (BPNN) in fig 1 are the two machine learning approaches were taken to build the forecast model. Just about seven years of data have taken comprising of every day open, high, low and closing prices, volume traded and value traded. The five-day time frame of the four RDP (relative difference in the percentage of price) values is utilized to determine the input values. EMA15 (Exponential Moving average) is utilized to keep the information of the actual closing price because when the application of RDP changing to the actual closing price may eliminate some useful data. Smoothing the closing price with 3-day EMA gives the output variable RDP +5 . Because the prediction performance of neural networks is improved when the application of smoothing change to the reliant variable. 


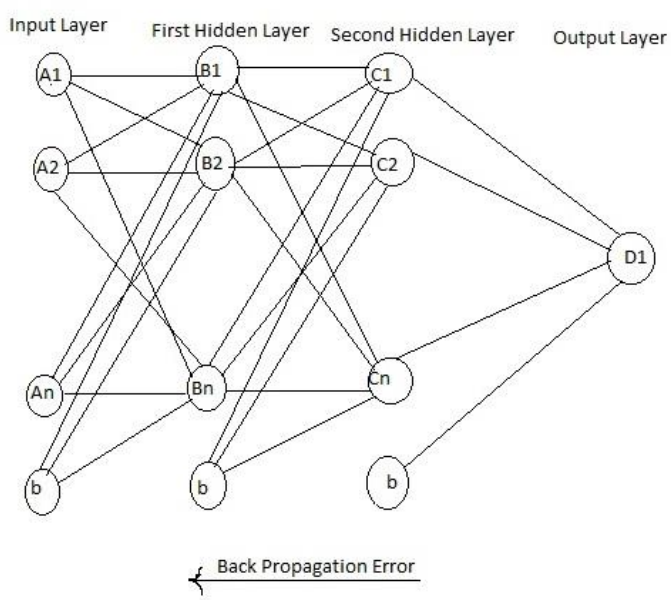

Fig.1: Back Propagation Neural Network

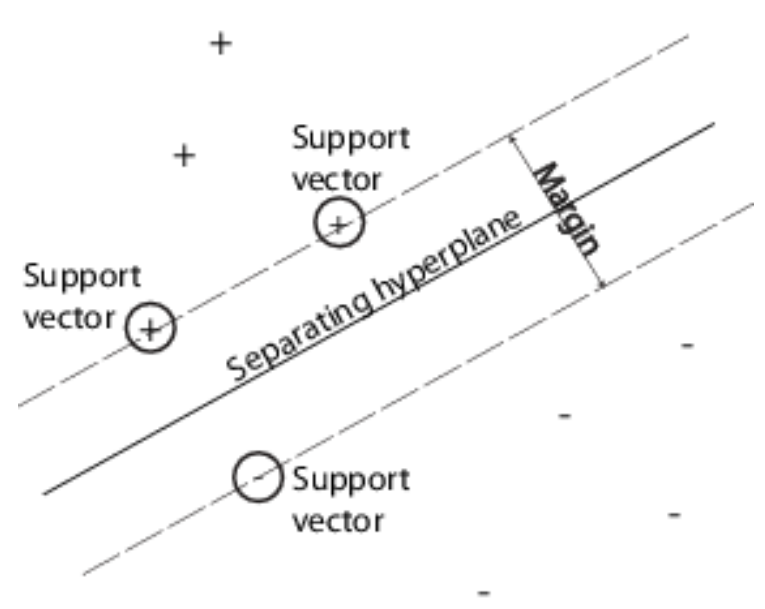

Fig .2: Support Vector Machine

Data scaling is the other technique utilized in this to scale the data points in the scope of $[-0.9 ; 0.9]$. Both positive and negative values are incorporated into the data points. The performance of prediction was assessed by utilizing statistical metrics, NMSE (Normalized Mean Square Error), DS (Directional symmetry), MAE (Mean Absolute error). The deviation between the real values and anticipated values are MAE and NMSE. After the training, based on the results, it is observed that SVM gives the best performance contrasted to BPNN in various aspects.

(Karathanasopoulos et al., 2016) Proposed ESVM (Evolutionary Support Vector Machine) as a stock predictor model. ESVM in Fig 3 is a combination of Support Vector Machines (SVMs) and Genetic Algorithms (GAs). In classification issues for data analysis and pattern recognition, SVMs are utilized. To enhance the parameters of SVM and to calculate the optimal feature subset GA is utilized. The model is proposed to avoid the overfitting by deploying the GA to modify the qualities of SVM model and to choose optimal inputs to achieve highest statistical and trade performance. The decision of the proposed framework is legitimized by its ability to wear down high-dimensional spaces, a similar number of information sources is seen as, its enhanced union properties and its ability reveal high beneficial trading systems. The ESVM is contrasted with five conventional methods and results acquired are extremely great as far as trading and statistical metrics. The ESVM conveys the phenomenal trading performance as far as annualized returns. It acquires the high simplification capacity of the SVM classifiers and this is the fundamental reason behind creating such a high trade performance in the out-of-test information collection while keeping the high trade performance in the dataset.

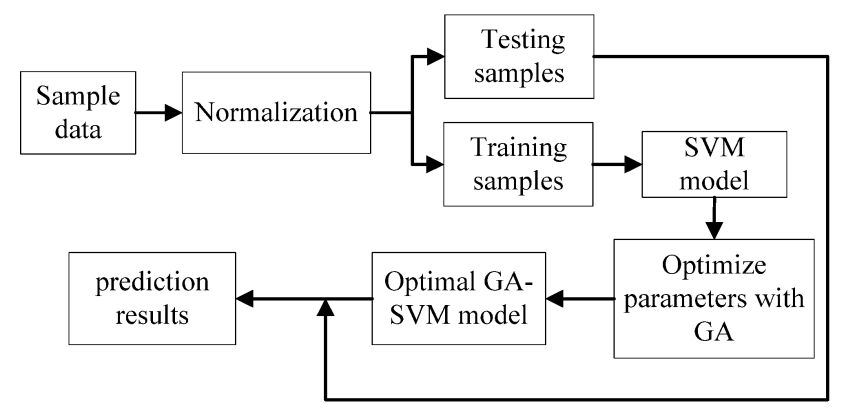

Fig.3: ESVM (Evolutionary Support Vector Machine)

(Lee, 2009) contrasted the SVM with BPNN as a target. Twenty future contracts are on commodities and nine future contracts are on spot indexes and eleven future contracts are in foreign currencies were taken as input data. The data set is partitioned into five gatherings out of which $80 \%$ of data is considered as training set and $20 \%$ of data is considered as a testing set. The scope of scale data is $(0,1)$. To standardize the feature component of the predetermined range direct scaling has to be done. It ensures the large esteem input traits don't overwhelm small esteem inputs; subsequently serves to decrease prediction errors. The Radial Basis Function (RBF) is utilized as a kernel function for SVM. The samples will be mapped to the high-dimensional space in a nonlinear way utilizing the RBF kernel which can deal with nonlinear issues. Cross-validation was done to stay away from the overfitting issues. When the results were compared in each feature selection SVM demonstrates the superb execution of BPNN for each test data.

(Rosillo, Giner, \& De La Fuente, 2014) considered Relative Strength Index (RSI) and Moving Average Convergence and divergence (MACD) as the inputs for SVM. RSI is for high cap stocks whereas MACD is for small cap stocks. An SVM classifier has been picked to make a quantitative decision. SVMs are extremely valuable for the investor while making decisions contrasted with other machine learning approaches. A time period of 200, 250,300 , and 500 days have been tested to out of which 250 days time period gave the best results. So the data of 250 days are considered as the training data out of which the values of 5 days ahead is considered in decision making whether to offer or purchase the stocks. The SVM first classifies the inputs and then tries to isolate it into two classes for purchasing and offering. HeavyTailed Radial Basis Function (HTRBF) is utilized as a kernel function for SVM. SVM algorithm is applied for making legitimate choices. With the end results when contrasted to other trading strategies it is observed that SVM outperforms in high volatility and low volatile whereas a bearish trend and bullish trend.

A variety of machine learning strategies have been utilized in recent period to foresee the stock price. Neural Network and support vector machine are the most usable among those SVM and NNs are both standard machine learning approaches to anticipate currency market data. Regression and classification are the two noteworthy applications of support vector machine. One of the real uniqueness of Support Vector Regression (SVR) is that as opposed to limiting the experiential training fault, SVR endeavors to reduce the generalized bound to achieve the best execution. This speculation mistake bound is the mix of the training fault and a regularization term that controls the difficulty of the difficulty space. (Kim, 2003), inspected the possibility of applying SVM into the currency market anticipating by contrasting it and backpropagation neural systems and case-based thinking. The test comes about demonstrating that SVM gives a promising contrasting option to currency market forecast. 


\section{Featured Work}

The support vector machine is the foremost practice for stock market prediction when compared with other techniques, but, even SVM has some limitations. Merging the SVM with some other model to produce an evolutionary technique for predicting the stock prices. That it will increase the exactness and accuracy of the prediction.

\section{Conclusion}

Currency markets play a prominent role in the business field. So the anticipation of the currency market is a challenging task for the researchers. There are several conventional models for foreseeing, yet those are less exact. The traditional models require a lot of data to anticipate. But the current trending model is Support Vector Machine, a machine learning approach, giving the best results with less data when contrasted with the conventional models and other machine learning approaches. SVM outperforms, however, the information is either linear or nonlinear. ESVM is a hybrid approach demonstrates the most elevated execution as far as annualized returns. The final conclusion of this study is SVM approach is the best strategy for anticipating stock market contrasted with other conventional models as well as other machine learning approaches.

\section{Acknowledgement}

My sincere thanks to all my previous authors. I have accomplished great learning in view of your work.

\section{References}

[1] Karathanasopoulos (2016). Stock market anticipation utilizing evolutionary support vector machines: an application of the ASE20 index. The European Journal of Finance, 22 (12), 11451163.

[2] Lee (2009). Utilizing support vector machine with a hybrid feature selection technique for the stock pattern forecast. Expert Systems with Applications, 36 (8), 10896-10904.

[3] PrasadDas (2012). Support Vector Machines at Prediction of Futures Costs in Indian Stock Market. International Journal of Computer Applications, 41 (3), 22-26.

[4] Rosillo (2014). Stock market simulation utilizing support vector machines. Journal of Forecasting, 33 (6), 488-500

[5] Kim, K. (2003). Financial time series forecasting using support vector machines. Neurocomputing, 55(1-2), 307-319. https://doi.org/10.1016/S0925-2312(03)00372-2

[6] Lai, L. K. C., \& Liu, J. N. K. (2010). Stock forecasting using support vector machine. Proceedings of the Ninth International Conference on Machine Learning and Cybernetics, (July), $1607-$ 1614 .

[7] Madge, S. (2015). Predicting Stock Price Direction using Support Vector Machines. Independent Work Report. Retrieved from

https://www.cs.princeton.edu/sites/default/files/uploads/saahil_m adge.pdf

[8] Meesad, P., \& Rasel, R. I. (2013). Predicting stock market price using support vector regression. 2013 International Conference on Informatics, Electronics, and Vision (ICIEV), (May), 1-6. https://doi.org/10.1109/ICIEV.2013.6572570

[9] Sap, M. N. M., \& Awan, a. M. (2005). Stock Market Prediction Using Support Vector Machines. Jurnal Teknologi Maklumat, Jilid 17,(July), 27-35.

[10] Shafiee, M., Yazdi, H. M., Panahi, H., \& Hesari, H. (2013) Forecasting Stock Returns using Support Vector Machine and Decision Tree: a Case Study in Iran Stock Exchange. International Journal of Economy, Management, and Social Sciences, 2(9), 746-751.

[11] Studies, E. (2000). A Stock Market Prediction Method Based on Support Vector Machines (SVM) and Independent Componen Analysis (ICA), 12-21.
[12] Abhishek, K., Khairwa, A., Pratap, T., \& Prakash, S. (2012). A stock market prediction model using Artificial Neural Network. 2012 Third International Conference on Computing, Communication and Networking Technologies (ICCCNT'12), (July), 1-5. https://doi.org/10.1109/ICCCNT.2012.6396089

[13] Iacomin, R. (2015). Stock market prediction. 2015 19th International Conference on System Theory, Control, and Computing (ICSTCC), 200-205. https://doi.org/10.1109/ICSTCC.2015.7321293

[14] Ichinose, K., \& Shimada, K. (2016). Stock market prediction from news on the web and a new evaluation approach in trading. Proceedings - 2016 5th IIAI International Congress on Advanced Applied Informatics, IIAI-AAI 2016, 77-81. https://doi.org/10.1109/IIAI-AAI.2016.157

[15] Parida, A., Kothari, S., \& Vineeth, N. (2015). International Journal of Advanced Research in Computer Science and Software Engineering, 5(4), 922-926.

[16] Porshnev, A., Redkin, I., \& Shevchenko, A. (2013). Machine learning in the prediction of stock market indicators based on historical data and data from twitter sentiment analysis. Proceedings - IEEE 13th International Conference on Data Mining Workshops, ICDMW 2013, 440-444. https://doi.org/10.1109/ICDMW.2013.111

[17] Rajput, V., \& Bobde, S. (2016). Stock Market Forecasting Techniques : Literature Survey, 5(6), 500-506.

[18] Wang, D., Liu, X., \& Wang, M. (2013). A DT-SVM strategy for stock futures prediction with big data. Proceedings - 16th IEEE International Conference on Computational Science and Engineering, CSE 2013, 1005-1012. https://doi.org/10.1109/CSE.2013.147

[19] Xing, T., Sun, Y., Wang, Q., \& Yu, G. (2013). The analysis and prediction of the stock price. 2013 IEEE International Conference on Granular Computing, GrC 2013, 368-373. https://doi.org/10.1109/GrC.2013.6740438

[20] Yetis, Y., Kaplan, H., \& Jamshidi, M. (2014). Stock market prediction by using the artificial neural network. 2014 World Automation Congress (WAC), 718-722. https://doi.org/10.1109/WAC.2014.6936118 